Bull. Korean Math. Soc. 51 (2014), No. 6, pp. 1863-1874

http://dx.doi.org/10.4134/BKMS.2014.51.6.1863

\title{
BOOST INVARIANT SURFACES WITH POINTWISE 1-TYPE GAUSS MAP IN MINKOWSKI 4-SPACE $\mathrm{E}_{1}^{4}$
}

\author{
Ferdag Kahraman Aksoyak and Yusuf Yayli
}

\begin{abstract}
In this paper, we study spacelike rotational surfaces which are called boost invariant surfaces in Minkowski 4-space $\mathbb{E}_{1}^{4}$. We give necessary and sufficient condition for flat spacelike rotational surface to have pointwise 1-type Gauss map. Also, we obtain a characterization for boost invariant marginally trapped surface with pointwise 1-type Gauss map.
\end{abstract}

\section{Introduction}

The notion of finite type mapping was introduced by B. Y. Chen in late 1970's. A smooth map $\phi$ from a compact Riemannian manifold $M$ into $m$ dimensional Euclidean space $\mathbb{E}^{m}$ is said to be of finite type if it is a finite sum of eigenfunctions of Laplacian $\Delta$ of $M$. More precisely, the smooth map $\phi: M \rightarrow \mathbb{E}^{m}$ is of finite type if it can be expressed as a finite sum

$$
\phi=\phi_{0}+\sum_{i=1}^{k} \phi_{i}
$$

where $\phi_{0}$ is a constant map, $\phi_{1}, \ldots, \phi_{k}$ are non-constant maps such that $\Delta \phi_{i}=$ $\lambda_{i} \phi_{i}, \lambda_{i} \in \mathbb{R}, i=1,2, \ldots, k$. If $\lambda_{1}, \lambda_{2}, \ldots, \lambda_{k}$ are all different, then the map $\phi$ is said to be of $k$-type.

Similarly, an isometric immersion $x$ of a submanifold in the Euclidean space $\mathbb{E}^{m}$ or pseudo-Euclidean space $\mathbb{E}_{s}^{m}$ with signature $(s, m-s)$ is said to be finite type, if each component of its position vector field $x$ can be written as a finite sum of eigenfunctions of the Laplacian $\Delta$ of $M$.

This notion of finite type immersions is naturally extended to differentiable maps of $M$ in particular, to Gauss maps of submanifolds [6] and many authors studied submanifolds with finite type Gauss map. If a submanifold $M$ of a Euclidean space or pseudo-Euclidean space has 1-type Gauss map $G$, then $G$ satisfies $\Delta G=\lambda(G+C)$ for some $\lambda \in \mathbb{R}$ and some constant vector $C$.

Received December 3, 2013.

2010 Mathematics Subject Classification. 53B25, 53C50.

Key words and phrases. rotation surface, Gauss map, pointwise 1-type Gauss map, marginally trapped surface, Minkowski space. 
However the Laplacian of the Gauss map of some typical well-known surfaces such as a helicoid, a catenoid and right cone in 3-dimensional Euclidean space $E^{3}$ and a helicoids of the 1st, 2nd and 3rd kind, conjugate Enneper's surface of the second kind and B-scrolls in 3-dimensional Minkowski space $E_{1}^{3}$ take a somewhat different form namely,

$$
\Delta G=f(G+C)
$$

for some non-zero smooth function $f$ on $M$ and some constant vector $C$. So a submanifold $M$ of a pseudo-Euclidean space $\mathbb{E}_{s}^{m}$ is said to have pointwise 1-type Gauss map if its Gauss map satisfies (1) for some smooth function $f$ on $M$ and some constant vector $C$. A pointwise 1-type Gauss map is called proper if the function $f$ defined by $(1)$ is non-constant. A submanifold with pointwise 1-type Gauss map is said to be of the first kind if the vector $C$ in (1) is zero vector. Otherwise, the pointwise 1-type Gauss map is said to be of the second kind.

Surfaces in Euclidean space and in pseudo-Euclidean space with pointwise 1-type Gauss map were recently studied in [3], [5], [7], [8], [9], [10], [11], [12], [14], [18], [21], [22]. Also Dursun and Turgay in [13] gave all general rotational surfaces in $\mathbb{E}^{4}$ with proper pointwise 1-type Gauss map of the first kind and classified minimal rotational surfaces with proper pointwise 1-type Gauss map of the second kind. Arslan et al. in [1] investigated rotational embedded surface with pointwise 1-type Gauss map. Arslan at el. in [2] gave necessary and sufficient conditions for Vranceanu rotation surface to have pointwise 1-type Gauss map. Yoon in [24] showed that flat Vranceanu rotation surface with pointwise 1-type Gauss map is a Clifford torus and in [23] studied rotation surfaces in the 4-dimensional Euclidean space with finite type Gauss map. Kim and Yoon in [19] obtained the complete classification theorems for the flat rotation surfaces with finite type Gauss map and pointwise 1-type Gauss map.

On the other hand, trapped surfaces, introduced by Penrose in 1965, have a fundamental role in the study of the singularity theorems in General Relativity. If the mean curvature vector of a surface in $E_{1}^{4}$ is timelike everywhere, it is called trapped surfaces; if the mean curvature vector is always null (the mean curvature vector is proportional to one of the null normals), the surface is called marginally trapped surface. Since the mean curvature of such spacelike surface $H$ satisfy $\|H\|=0$, in mathematical literature these surfaces are called quasiminimal. In general relativity, marginally trapped surfaces are used the study of the surfaces of black hole.

S. Haesen and M. Ortega in [16] and [17] classified marginally trapped surfaces which are invariant under a spacelike rotations and boost transformations in Minkowski 4-space. Also B. Y. Chen classify marginally trapped Lorentzian flat surfaces and biharmonic surfaces in the Pseudo Euclidean space $E_{2}^{4}$ [4]. Milousheva in [20] studied marginally trapped surface with pointwise 1-type Gauss map in Minkowski 4-space and proved that marginally trapped surface 
is of pointwise 1-type Gauss map if and only if it has parallel mean curvature vector field.

In this paper, we study spacelike surfaces which are invariant under boost transformation (hyperbolic rotations) in Minkowski 4-space. We give necessary and sufficient condition for flat spacelike rotational surface to have pointwise 1-type Gauss map. Also we obtain a characterization for boost invariant marginally trapped surface with pointwise 1-type Gauss map.

\section{Preliminaries}

Let $E_{s}^{m}$ be the $m$-dimensional pseudo-Euclidean space with signature $(s, m-$ $s)$. Then the metric tensor $g$ in $E_{s}^{m}$ has the form

$$
g=\sum_{i=1}^{m-s}\left(d x_{i}\right)^{2}-\sum_{i=m-s+1}^{m}\left(d x_{i}\right)^{2},
$$

where $\left(x_{1}, \ldots, x_{m}\right)$ is a standard rectangular coordinate system in $E_{s}^{m}$.

Let $M$ be an $n$-dimensional pseudo-Riemannian submanifold of a $m$-dimensional pseudo-Euclidean space $\mathbb{E}_{s}^{m}$. We denote Levi-Civita connections of $\mathbb{E}_{s}^{m}$ and $M$ by $\tilde{\nabla}$ and $\nabla$, respectively. Let $e_{1}, \ldots, e_{n}, e_{n+1}, \ldots, e_{m}$ be an adapted local orthonormal frame in $\mathbb{E}_{s}^{m}$ such that $e_{1}, \ldots, e_{n}$ are tangent to $M$ and $e_{n+1}, \ldots, e_{m}$ normal to $M$. We use the following convention on the ranges of indices: $1 \leq i, j, k, \ldots \leq n, n+1 \leq r, s, t, \ldots \leq m, 1 \leq A, B, C, \ldots \leq m$.

Let $\omega_{A}$ be the dual-1 form of $e_{A}$ defined by $\omega_{A}(X)=\left\langle e_{A}, X\right\rangle$ and $\varepsilon_{A}=$ $\left\langle e_{A}, e_{A}\right\rangle= \pm 1$. Also, the connection forms $\omega_{A B}$ are defined by

$$
d e_{A}=\sum_{B} \varepsilon_{B} \omega_{A B} e_{B}, \quad \omega_{A B}+\omega_{B A}=0 .
$$

Then we have

$$
\tilde{\nabla}_{e_{k}}^{e_{i}}=\sum_{j=1}^{n} \varepsilon_{j} \omega_{i j}\left(e_{k}\right) e_{j}+\sum_{r=n+1}^{m} \varepsilon_{r} h_{i k}^{r} e_{r}
$$

and

$$
\tilde{\nabla}_{e_{k}}^{e_{s}}=-\sum_{j=1}^{n} \varepsilon_{j} h_{k j}^{s} e_{j}+\sum_{r=n+1}^{m} \varepsilon_{r} \omega_{s r}\left(e_{k}\right) e_{r},
$$

where $h_{i k}^{r}$ the coefficients of the second fundamental form $h$. The mean curvature vector $H$ of $M$ in $\mathbb{E}_{s}^{m}$ is defined by

$$
H=\frac{1}{n} \sum_{s=n+1}^{m} \sum_{i=1}^{n} \varepsilon_{i} \varepsilon_{s} h_{i i}^{s} e_{s}
$$

and the Gaussian curvature $K$ of $M$ is given by

$$
K=\sum_{s=n+1}^{m} \varepsilon_{s}\left(h_{11}^{s} h_{22}^{s}-h_{12}^{s} h_{21}^{s}\right) .
$$


Also normal curvature tensor $R^{D}$ of $M$ in $\mathbb{E}_{s}^{m=n+2}$ is given by

$$
R^{D}\left(e_{j}, e_{k} ; e_{r}, e_{s}\right)=\sum_{i=1}^{n} \varepsilon_{i}\left(h_{i k}^{r} h_{i j}^{s}-h_{i j}^{r} h_{i k}^{s}\right) .
$$

We recall that a surface $M$ in $\mathbb{E}_{1}^{4}$ is called extremal surface if its mean curvature vector vanishes. If its Gaussian curvature vanishes, the surface $M$ is called flat surface. If its normal curvature tensor $R^{D}$ vanishes identically, then a surface $M$ in $\mathbb{E}_{1}^{4}$ is said to have flat normal bundle.

For any real function $f$ on $M$ the Laplacian $\Delta f$ of $f$ is given by

$$
\Delta f=-\varepsilon_{i} \sum_{i}\left(\tilde{\nabla}_{e_{i}} \tilde{\nabla}_{e_{i}} f-\tilde{\nabla}_{\nabla_{e_{i}}^{e_{i}}} f\right) .
$$

Let us now define the Gauss map $G$ of a submanifold $M$ into $G(n, m)$ in $\wedge^{n} \mathbb{E}_{s}^{m}$, where $G(n, m)$ is the Grassmannian manifold consisting of all oriented $n$-planes through the origin of $\mathbb{E}_{s}^{m}$ and $\wedge^{n} \mathbb{E}_{s}^{m}$ is the vector space obtained by the exterior product of $n$ vectors in $\mathbb{E}_{s}^{m}$. Let $e_{i_{1}} \wedge \cdots \wedge e_{i_{n}}$ and $f_{j_{1}} \wedge \cdots \wedge f_{j_{n}}$ be two vectors of $\wedge^{n} \mathbb{E}_{s}^{m}$, where $\left\{e_{1}, \ldots, e_{m}\right\}$ and $\left\{f_{1}, \ldots, f_{m}\right\}$ are orthonormal bases of $\mathbb{E}_{s}^{m}$. Define an indefinite inner product $\langle$,$\rangle on \wedge^{n} \mathbb{E}_{s}^{m}$ by

$$
\left\langle e_{i_{1}} \wedge \cdots \wedge e_{i_{n}}, f_{j_{1}} \wedge \cdots \wedge f_{j_{n}}\right\rangle=\operatorname{det}\left(\left\langle e_{i_{l}}, f_{j_{k}}\right\rangle\right) .
$$

Therefore, for some positive integer $t$, we may identify $\wedge^{n} \mathbb{E}_{s}^{m}$ with some Euclidean space $\mathbb{E}_{t}^{N}$ where $N=\left(\begin{array}{c}m \\ n\end{array}\right)$. The map $G: M \rightarrow G(n, m) \subset E_{t}^{N}$ defined by $G(p)=\left(e_{1} \wedge \cdots \wedge e_{n}\right)(p)$ is called the Gauss map of $M$, that is, a smooth map which carries a point $p$ in $M$ into the oriented $n$-plane in $\mathbb{E}_{s}^{m}$ obtained from parallel translation of the tangent space of $M$ at $p$ in $\mathbb{E}_{s}^{m}$.

\section{Boost invariant surfaces with pointwise 1-type Gauss map in $E_{1}^{4}$}

In this section, we consider spacelike surfaces in the Minkowski space $E_{1}^{4}$ which are invariant under the following subgroup of direct, linear isometries of $E_{1}^{4}$ :

$$
G=\left\{\left(\begin{array}{cccc}
\cosh t & \sinh t & 0 & 0 \\
\sinh t & \cosh t & 0 & 0 \\
0 & 0 & 1 & 0 \\
0 & 0 & 0 & 1
\end{array}\right): t \in \mathbb{R}\right\}
$$

well-known as boost isometries [16].

$$
\varphi(t, s)=\left(\begin{array}{cccc}
\cosh t & \sinh t & 0 & 0 \\
\sinh t & \cosh t & 0 & 0 \\
0 & 0 & 1 & 0 \\
0 & 0 & 0 & 1
\end{array}\right)\left(\begin{array}{c}
\alpha_{1}(s) \\
0 \\
\alpha_{3}(s) \\
\alpha_{4}(s)
\end{array}\right)
$$

$$
M: \varphi(t, s)=\left(\alpha_{1}(s) \cosh t, \alpha_{1}(s) \sinh t, \alpha_{3}(s), \alpha_{4}(s)\right),
$$


where the profile curve of $M$ is unit speed spacelike curve, that is, $-\left(\alpha_{1}^{\prime}(s)\right)^{2}+$ $\left(\alpha_{3}^{\prime}(s)\right)^{2}+\left(\alpha_{4}^{\prime}(s)\right)^{2}=1$. We choose a moving frame $e_{1}, e_{2}, e_{3}, e_{4}$ such that $e_{1}, e_{2}$ are tangent to $M$ and $e_{3}, e_{4}$ are normal to $M$ which are given by the following:

$$
\begin{aligned}
e_{1}= & \left(\alpha_{1}^{\prime}(s) \cosh t, \alpha_{1}^{\prime}(s) \sinh t, \alpha_{3}^{\prime}(s), \alpha_{4}^{\prime}(s)\right) \\
e_{2}= & (\sinh t, \cosh t, 0,0) \\
e_{3}= & \frac{1}{\sqrt{1+\left(\alpha_{1}^{\prime}(s)\right)^{2}}}\left(\left(1+\left(\alpha_{1}^{\prime}(s)\right)^{2}\right) \cosh t,\left(1+\left(\alpha_{1}^{\prime}(s)\right)^{2}\right) \sinh t\right. \\
& \left.\alpha_{1}^{\prime}(s) \alpha_{3}^{\prime}(s), \alpha_{1}^{\prime}(s) \alpha_{4}^{\prime}(s)\right) \\
e_{4}= & \frac{1}{\sqrt{1+\left(\alpha_{1}^{\prime}(s)\right)^{2}}}\left(0,0,-\alpha_{4}^{\prime}(s), \alpha_{3}^{\prime}(s)\right) .
\end{aligned}
$$

Then it is easily seen that

$$
\left\langle e_{1}, e_{1}\right\rangle=\left\langle e_{2}, e_{2}\right\rangle=\left\langle e_{4}, e_{4}\right\rangle=1,\left\langle e_{3}, e_{3}\right\rangle=-1
$$

we have the dual 1-forms as:

$$
\omega_{1}=d s \quad \text { and } \quad \omega_{2}=\alpha_{1}(s) d t .
$$

By a direct computation we have components of the second fundamental form and the connection forms as:

$$
\begin{aligned}
& h_{11}^{3}=-c(s), h_{12}^{3}=0, \quad h_{22}^{3}=-b(s), \\
& h_{11}^{4}=d(s), \quad h_{12}^{4}=0, \quad h_{22}^{4}=0, \\
\omega_{12}= & a(s) b(s) \omega_{2}, \quad \omega_{13}=-c(s) \omega_{1}, \quad \omega_{14}=d(s) \omega_{1}, \\
\omega_{23}= & -b(s) \omega_{2}, \quad \omega_{24}=0, \quad \omega_{34}=a(s) d(s) \omega_{1} .
\end{aligned}
$$

By covariant differentiation with respect to $e_{1}$ and $e_{2}$ a straightforward calculation gives:

$$
\begin{aligned}
\tilde{\nabla}_{e_{1}} e_{1} & =c(s) e_{3}+d(s) e_{4}, \\
\tilde{\nabla}_{e_{2}} e_{1} & =a(s) b(s) e_{2}, \\
\tilde{\nabla}_{e_{1}} e_{2} & =0 \\
\tilde{\nabla}_{e_{2}} e_{2} & =-a(s) b(s) e_{1}+b(s) e_{3}, \\
\tilde{\nabla}_{e_{1}} e_{3} & =c(s) e_{1}+a(s) d(s) e_{4}, \\
\tilde{\nabla}_{e_{2}} e_{3} & =b(s) e_{2}, \\
\tilde{\nabla}_{e_{1}} e_{4} & =-d(s) e_{1}+a(s) d(s) e_{3}, \\
\tilde{\nabla}_{e_{2}} e_{4} & =0
\end{aligned}
$$

where

$$
a(s)=\frac{\alpha_{1}^{\prime}(s)}{\sqrt{1+\left(\alpha_{1}^{\prime}(s)\right)^{2}}},
$$




$$
\begin{gathered}
b(s)=\frac{\sqrt{1+\left(\alpha_{1}^{\prime}(s)\right)^{2}}}{\alpha_{1}(s)}, \\
c(s)=\frac{\alpha_{1}^{\prime \prime}(s)}{\sqrt{1+\left(\alpha_{1}^{\prime}(s)\right)^{2}}}, \\
d(s)=\frac{-\alpha_{3}^{\prime \prime}(s) \alpha_{4}^{\prime}(s)+\alpha_{4}^{\prime \prime}(s) \alpha_{3}^{\prime}(s)}{\sqrt{1+\left(\alpha_{1}^{\prime}(s)\right)^{2}}} .
\end{gathered}
$$

The Gaussian curvature $K$ of $M$ is given by

$$
K=-b(s) c(s) \text {. }
$$

The mean curvature $H$ of $M$ is given by

$$
H=\frac{1}{2}\left(-h_{1} e_{3}+h_{2} e_{4}\right), \quad h_{1}=-(b+c) \text { and } h_{2}=d .
$$

By using (3), (8) and straight-forward computation, the Laplacian $\Delta G$ of the Gauss map $G$ can be expressed as

$$
\Delta G=A(s)\left(e_{1} \wedge e_{2}\right)+B(s)\left(e_{2} \wedge e_{3}\right)+D(s)\left(e_{2} \wedge e_{4}\right),
$$

where

$$
\begin{gathered}
A(s)=d^{2}(s)-b^{2}(s)-c^{2}(s), \\
B(s)=b^{\prime}(s)+c^{\prime}(s)+a(s) d^{2}(s), \\
D(s)=d^{\prime}(s)+a(s) d(s)(b(s)+c(s)) .
\end{gathered}
$$

Theorem 1. Let $M$ be the flat rotation surface given by the parametrization (4). Then $M$ has pointwise 1-type Gauss map if and only if the profile curve of $M$ is parametrized by

$$
\begin{aligned}
& \alpha_{1}(s)=a_{1}, \\
& \alpha_{3}(s)=\frac{1}{a_{2}} \sin \left(a_{2} s+a_{3}\right), \\
& \alpha_{4}(s)=-\frac{1}{a_{2}} \cos \left(a_{2} s+a_{3}\right),
\end{aligned}
$$

$$
\begin{aligned}
& \alpha_{1}(s)=b_{1} s+b_{2}, \\
& \alpha_{3}(s)=\int\left(1+b_{1}^{2}\right)^{\frac{1}{2}} \cos \left(b \ln \left|b_{1} s+b_{2}\right|\right) d s, \\
& \alpha_{4}(s)=\int\left(1+b_{1}^{2}\right)^{\frac{1}{2}} \sin \left(b \ln \left|b_{1} s+b_{2}\right|\right) d s,
\end{aligned}
$$

where $a_{1}, a_{2}, a_{3} b_{1} \neq 0, b_{2}, b_{3}$ and $b=\frac{b_{3}}{b_{1}\left(1+b_{1}^{2}\right)^{\frac{1}{2}}}$ are real constants. 
Proof. Let $M$ be the flat rotation surface given by the parametrization (4). We suppose that $M$ has pointwise 1-type Gauss map. By using (1) and (15), we have

$$
\begin{aligned}
f+f\left\langle C, e_{1} \wedge e_{2}\right\rangle & =A(s), \\
f\left\langle C, e_{2} \wedge e_{3}\right\rangle & =-B(s), \\
f\left\langle C, e_{2} \wedge e_{4}\right\rangle & =D(s),
\end{aligned}
$$

and

$$
\left\langle C, e_{1} \wedge e_{3}\right\rangle=\left\langle C, e_{1} \wedge e_{4}\right\rangle=\left\langle C, e_{3} \wedge e_{4}\right\rangle=0
$$

By differentiating (22) covariantly with respect to $e_{2}$, we have

$$
\begin{aligned}
-a(s) B(s)+A(s)-f & =0 \\
a(s) D(s) & =0 \\
D(s) & =0 .
\end{aligned}
$$

In this case, firstly, we assume that $a(s)=0$ and $D(s)=0$. From (9), we obtain that $\alpha_{1}(s)=a_{1}$. Since the profile curve is unit speed spacelike curve, we can write $\left(\alpha_{3}^{\prime}(s)\right)^{2}+\left(\alpha_{4}^{\prime}(s)\right)^{2}=1$. Also we can put

$$
\alpha_{3}^{\prime}(s)=\cos \theta(s) \text { and } \alpha_{4}^{\prime}(s)=\sin \theta(s),
$$

where $\theta$ is smooth angle function. On the other hand, since $D(s)=0$, from (18) we obtain as

$$
d(s)=a_{2}, \quad a_{2} \text { is non zero constant. }
$$

By using (12), (23) and (24) we get

$$
\theta(s)=a_{2} s+a_{3} .
$$

So from (23) and (25) we have

$$
\begin{aligned}
\alpha_{3}(s) & =\frac{1}{a_{2}} \sin \left(a_{2} s+a_{3}\right), \\
\alpha_{4}(s) & =-\frac{1}{a_{2}} \cos \left(a_{2} s+a_{3}\right) .
\end{aligned}
$$

Now we assume that $a(s) \neq 0$ and $D(s)=0$. Since $M$ is flat, (11) and (13) imply that

$$
\alpha_{1}(s)=b_{1} s+b_{2}
$$

for some constants $b_{1} \neq 0$ and $b_{2}$. Since the profile curve is unit speed spacelike curve, we can write $\left(\alpha_{3}^{\prime}(s)\right)^{2}+\left(\alpha_{4}^{\prime}(s)\right)^{2}=1+b_{1}^{2}$. Also we can put

$$
\begin{aligned}
& \alpha_{3}^{\prime}(s)=\left(1+b_{1}^{2}\right)^{\frac{1}{2}} \cos \theta(s), \\
& \alpha_{4}^{\prime}(s)=\left(1+b_{1}^{2}\right)^{\frac{1}{2}} \sin \theta(s),
\end{aligned}
$$


where $\theta$ is smooth angle function. By using (9), (10) and (18), we get

$$
d(s)=\frac{b_{3}}{b_{1} s+b_{2}} .
$$

On the other hand, by using (12), (26) and (27) we have

$$
d(s)=\left(1+b_{1}^{2}\right)^{\frac{1}{2}} \theta^{\prime}(s) .
$$

By combining (28) and (29) we obtain

$$
\theta(s)=b \ln \left|b_{1} s+b_{2}\right|,
$$

where $b=\frac{b_{3}}{b_{1}\left(1+b_{1}^{2}\right)^{\frac{1}{2}}}$. So by substituting (30) into (27) we can write

$$
\begin{aligned}
& \alpha_{3}(s)=\int\left(1+b_{1}^{2}\right)^{\frac{1}{2}} \cos \left(b \ln \left|b_{1} s+b_{2}\right|\right) d s, \\
& \alpha_{4}(s)=\int\left(1+b_{1}^{2}\right)^{\frac{1}{2}} \sin \left(b \ln \left|b_{1} s+b_{2}\right|\right) d s .
\end{aligned}
$$

Conversely, the surface $M$ which is parametrized by (19) and (20) is pointwise 1-type Gauss map for

$$
f(s)=-a(s) b^{\prime}(s)-a^{2}(s) d^{2}(s)+d^{2}(s)-b^{2}(s)
$$

and

$$
C(s)=\frac{a(s) b^{\prime}(s)+a^{2}(s) d^{2}(s)}{f(s)}\left(e_{1} \wedge e_{2}\right)+\frac{b^{\prime}(s)+a(s) d^{2}(s)}{f(s)}\left(e_{2} \wedge e_{3}\right),
$$

where it can be easily seen that $e_{1}(C(s))=0$ and $e_{2}(C(s))=0$. This completes the proof.

Corollary 1. Let $M$ be the flat rotation surface given by the parametrization (4). If $M$ has pointwise 1-type Gauss map, then the profile curve of $M$ is a circle or helix curve.

We will also use the following theorems and corollary.

Theorem 2 ([15]). Let $M$ be an oriented maximal surface in the Minkowski space $E_{1}^{4}$. Then $M$ has pointwise 1-type Gauss map of the first kind if and only if $M$ has flat normal bundle. Hence the Gauss map G satisfies (1) for $f=\|h\|^{2}$ and $C=0$, where $\|h\|^{2}$ is the squared length of the second fundamental form.

Theorem 3 ([16]). Let $M$ be a spacelike rotational surface in Minkowski 4space given by the parametrization (4). If $M$ marginally trapped surface, then

$$
\begin{aligned}
& \alpha_{3}(s)=\int\left(1+\left(\alpha_{1}^{\prime}\right)^{2}\right)^{\frac{1}{2}} \cos \theta(s) d s, \\
& \alpha_{4}(s)=\int\left(1+\left(\alpha_{1}^{\prime}\right)^{2}\right)^{\frac{1}{2}} \sin \theta(s) d s,
\end{aligned}
$$


and

$$
\theta(s)=-\epsilon \int \frac{1+\left(\alpha_{1}^{\prime}\right)^{2}+\alpha_{1}^{\prime} \alpha_{1}^{\prime \prime}}{\alpha_{1}\left(1+\left(\alpha_{1}^{\prime}\right)^{2}\right)^{\frac{1}{2}}}
$$

where $\epsilon= \pm 1$

Corollary 2 ([16]). Let $M$ be a spacelike rotational surface in Minkowski 4space given by the parametrization (4). If $M$ is a extremal surface, then a unit profile curve is given by

$$
\alpha(s)=\left(f(s), 0, \cos \zeta_{0} \sqrt{a_{1}} \arctan \left(\frac{s+a_{2}}{f(s)}\right), \sin \zeta_{0} \sqrt{a_{1}} \arctan \left(\frac{s+a_{2}}{f(s)}\right)\right),
$$

where $f(s)=\sqrt{a_{1}-\left(s+a_{2}\right)^{2}}$ and $a_{1}, a_{2}, \zeta_{0} \in \mathbb{R}, a_{1}>0$, being integration constants. In particular, the surface $M$ is immersed in a totally geodesic Lorentzian 3-space.

Theorem 4. Let $M$ be the marginally trapped surface given by the parametrization (4) in Minkowski 4-space. Then $M$ has pointwise 1-type Gauss map if and only if the profile curve is given by

$$
\begin{aligned}
\alpha_{1}(s) & =\left(\lambda_{1}-1\right)^{\frac{1}{2}}\left(u^{2}(s)+\lambda\right)^{\frac{1}{2}}, \\
\alpha_{3}(s) & =\int\left(\frac{\lambda_{1} u^{2}+\lambda}{u^{2}+\lambda}\right)^{\frac{1}{2}} \cos \theta(s) d s, \\
\alpha_{4}(s) & =\int\left(\frac{\lambda_{1} u^{2}+\lambda}{u^{2}+\lambda}\right)^{\frac{1}{2}} \sin \theta(s) d s,
\end{aligned}
$$

and

$$
\theta(s)=-\epsilon \frac{\lambda_{1}}{\left(\lambda_{1}-1\right)^{\frac{1}{2}}} \int \frac{\left(u^{2}+\lambda\right)^{\frac{1}{2}}}{\lambda_{1} u^{2}+\lambda} d s,
$$

where $u(s)=\delta s+\lambda_{3}, \lambda=\frac{\lambda_{2}}{\lambda_{1}-1}, \lambda_{1}, \lambda_{2}, \lambda_{3}, a_{1}$ and $a_{2}$ are real constants.

Proof. Let $M$ be marginally trapped surface. This means $\|H\|=0$ that is $\langle H, H\rangle=0$. By using (14), we get

$$
-(b(s)+c(s))=\epsilon d(s)
$$

where $\epsilon= \pm 1$. In this case, by using (34) and (15) we can rewrite the Laplacian $\Delta G$ of the Gauss map $G$ as

$$
\Delta G=A(s)\left(e_{1} \wedge e_{2}\right)-\epsilon N(s)\left(e_{2} \wedge e_{3}\right)+N(s)\left(e_{2} \wedge e_{4}\right),
$$

where

$$
N(s)=d^{\prime}(s)-\epsilon a(s) d^{2}(s) .
$$

We assume that $M$ has pointwise 1-type Gauss map. Then we have

$$
f+f\left\langle C, e_{1} \wedge e_{2}\right\rangle=A(s),
$$




$$
\begin{aligned}
& f\left\langle C, e_{2} \wedge e_{3}\right\rangle=\epsilon N(s), \\
& f\left\langle C, e_{2} \wedge e_{4}\right\rangle=N(s),
\end{aligned}
$$

and

$$
\left\langle C, e_{1} \wedge e_{3}\right\rangle=\left\langle C, e_{1} \wedge e_{4}\right\rangle=\left\langle C, e_{3} \wedge e_{4}\right\rangle=0 .
$$

By differentiating (38) with respect to $e_{2}$, we have

$$
\begin{aligned}
\epsilon a(s) N(s)+A(s)-f & =0 \\
a(s) N(s) & =0 \\
N(s) & =0 .
\end{aligned}
$$

In this case, firstly, we assume that $a(s)=0$ and $N(s)=0$. From (9) and (11), we obtain that $\alpha_{1}(s)=a_{1}$ and $c(s)=0$, respectively. Hence from (34) we get

$$
-b(s)=\epsilon d(s) .
$$

By using (39) and (16) we obtain that $A(s)=0$. So we have that $f=0$. This is a contradiction.

Now we assume that $a(s) \neq 0$ and $N(s)=0$. By combining (9), (10), (11), (12), (34) and (36), we obtain a differential equation as follows:

$$
\left(1+\left(\alpha_{1}^{\prime}(s)\right)^{2}+\alpha_{1}^{\prime}(s) \alpha_{1}^{\prime \prime}(s)\right)^{\prime} \alpha_{1}(s)\left(1+\left(\alpha_{1}^{\prime}(s)\right)^{2}\right)=0 .
$$

Since $\alpha_{1}>0$ and $1+\left(\alpha_{1}^{\prime}(s)\right)^{2} \neq 0$ we have

$$
1+\left(\alpha_{1}^{\prime}(s)\right)^{2}+\alpha_{1}^{\prime}(s) \alpha_{1}^{\prime \prime}(s)=\lambda_{1}
$$

whose the solition

$$
\alpha_{1}(s)=\left(\lambda_{1}-1\right)^{\frac{1}{2}}\left(u^{2}+\lambda\right)^{\frac{1}{2}} .
$$

By using (32) and (40) we get

$$
\theta(s)=-\epsilon \mu \int \frac{\left(u^{2}+\lambda\right)^{\frac{1}{2}}}{\lambda_{1} u^{2}+\lambda} d s,
$$

where $\delta= \pm 1, u(s)=\delta s+\lambda_{3}, \lambda=\frac{\lambda_{2}}{\left(\lambda_{1}-1\right)^{2}}$ and $\mu=\frac{\lambda_{1}}{\left(\lambda_{1}-1\right)^{\frac{1}{2}}}$.

Conversely, the surface $M$ which is parametrized by (33) has pointwise 1type Gauss map with

$$
f(s)=2 b(s) c(s)
$$

and

$$
C(s)=0 .
$$

This completes the proof.

Corollary 3. Let $M$ be marginally trapped surface given by the parametrization (4) in Minkowski 4-space. Then $M$ has pointwise 1-type Gauss map then $M$ is pointwise 1-type Gauss map of the first kind. 
Corollary 4. Let $M$ be a spacelike rotational surface in Minkowski 4-space given by the parametrization (4). If $M$ is extremal surface, then $M$ has pointwise 1-type Gauss map of the first kind.

Proof. We assume that $M$ is a spacelike rotational surface given by the parametrization (4). In that case by using (2) and (6) we obtain that $M$ has flat normal bundle. Hence from Theorem (2) if $M$ is extremal surface, then $M$ has pointwise 1-type Gauss map of the first kind.

\section{Conclusion}

In this paper, we study spacelike surfaces which are invariant under boost transformation (hyperbolic rotations) in Minkowski 4-space. We give necessary and sufficient condition for flat spacelike rotational surface to have pointwise 1-type Gauss map and we obtain that the profile curve of flat boost invariant surfaces with pointwise 1-type Gauss map is a circle or a helix curve. On the other hand, trapped surfaces, introduced by Penrose in 1965, have a fundamental role in the study of the singularity theorems in General Relativity and marginally trapped surfaces are used the study of the surfaces of black hole. Also we give a characterization for boost invariant marginally trapped surface with pointwise 1-type Gauss map.

\section{References}

[1] K. Arslan, B. K. Bayram, B. Bulca, Y. H. Kim, C. Murathan, and G. Öztürk, Rotational embeddings in $E^{4}$ with pointwise 1-type Gauss map, Turkish J. Math. 35 (2011), no. 3, 493-499.

[2] K. Arslan, B. K. Bayram, Y. H. Kim, C. Murathan, and G. Öztürk, Vranceanu surface in $E^{4}$ with pointwise 1-type Gauss map, Indian J. Pure. Appl. Math. 42 (2011), no. 1, 41-51.

[3] K. Arslan, B. Bulca, B. Kılıç, Y. H. Kim, C. Murathan, and G. Öztürk, Tensor product surfaces with pointwise 1-type Gauss map. Bull. Korean Math. Soc. 48 (2011), no. 3, 601-609.

[4] B. Y. Chen, Classification of marginally trapped Lorentzian flat surfaces in $E_{2}^{4}$ and its application to biharmonic surfaces, J. Math. Anal. Appl. 340 (2008), no. 2, 861-875.

[5] B. Y. Chen, M. Choi, and Y. H. Kim, Surfaces of revolution with pointwise 1-type Gauss map, J. Korean Math. 42 (2005), no. 3, 447-455.

[6] B. Y. Chen and P. Piccinni, Submanifolds with finite type-Gauss map, Bull. Austral. Math. Soc. 35 (1987), no. 2, 161-186.

[7] M. Choi and Y. H. Kim, Characterization of the helicoid as ruled surfaces with pointwise 1-type Gauss map, Bull. Korean Math. Soc. 38 (2001), no. 4, 753-761.

[8] M. Choi, D. S. Kim, and Y. H. Kim, Helicoidal surfaces with pointwise 1-type Gauss map, J. Korean Math. Soc. 46 (2009), no. 1, 215-223.

[9] M. Choi, Y. H. Kim, and D. W. Yoon, Classification of ruled surfaces with pointwise 1type Gauss map in Minkowski 3-space, Taiwanese J. Math. 15 (2011), no. 3, 1141-1161.

[10] U. Dursun, Hypersurfaces with pointwise 1-type Gauss map in Lorentz-Minkowski space, Proc. Est. Acad. Sci. 58 (2009), no. 3, 146-161.

[11] U. Dursun and G. G. Arsan, Surfaces in the Euclidean space $E^{4}$ with pointwise 1-type Gauss map, Hacet. J. Math. Stat. 40 (2011), no. 5, 617-625. 
[12] U. Dursun and E. Coşkun, Flat surfaces in the Minkowski space $E_{1}^{3}$ with pointwise 1-type Gauss map, Turkish J. Math. 36 (2012), no. 4, 613-629.

[13] U. Dursun and N. C. Turgay, General rotational surfaces in Euclidean space $E^{4}$ with pointwise 1-type Gauss map, Math. Commun. 17 (2012), no. 1, 71-81.

[14] _ On spacelike surfaces in Minkowski 4-space with pointwise 1-type Gauss map of second type, Balkan J. Geom. Appl. 17 (2012), no. 2, 34-45.

[15] _ Space-like surfaces in Minkowski space $E_{1}^{4}$ with pointwise 1-type Gauss map, arXiv:1305.5419.

[16] S. Haesen and M. Ortega, Boost invariant marginally trapped surfaces in Minkowski 4-space, Classical Quantum Gravity 24 (2007), no. 22, 5441-5452.

[17] _ Marginally trapped surfaces in Minkowski 4-space invariant under a rotation subgroup of the Lorentz group, Gen. Relativity Gravitation 41 (2009), no. 8, 1819-1834.

[18] Y. H. Kim and D. W. Yoon, Ruled surfaces with pointwise 1-type Gauss map, J. Geom. Phys. 34 (2000), no. 3-4, 191-205.

[19] Classification of rotation surfaces in pseudo Euclidean space, J. Korean Math. 41 (2004), no. 2, 379-396.

[20] V. Milousheva, Marginally trapped surfaces with pointwise 1-type Gauss map in Minkowski 4-space, Int. J. Geom. 2 (2013), no. 1, 34-43.

[21] A. Niang, On rotation surfaces in the Minkowski 3-dimensional space with pointwise 1-type Gauss map, J. Korean Math. Soc. 41 (2004), no. 6, 1007-1021.

$[22]$, Rotation surfaces with 1-type Gauss map, Bull. Korean Math. Soc. 42 (2005), no. 1, 23-27.

[23] D. W. Yoon, Rotation surfaces with finite type Gauss map in $E^{4}$, Indian J. Pure. Appl. Math. 32 (2001), no. 12, 1803-1808.

[24] Some properties of the Clifford torus as rotation surface, Indian J. Pure. Appl. Math. 34 (2003), no. 6, 907-915.

Ferdag Kahraman Aksoyak

Division of Elementary Mathematics Education

Ahi Evran University

KIRŞEHIR, TURKEY

E-mail address: ferda@erciyes.edu.tr

YusuF YAYLI

Department of MATHEMATiCs

ANKARA UNIVERSITY

ANKARA, TURKEY

E-mail address: yayli@science.ankara.edu.tr 